\title{
Limited Progress in Increasing Coverage of Neonatal and Child-health Interventions in Africa and Asia
}

\author{
Cynthia Boschi-Pinto, Rajiv Bahl, and José Martines \\ Newborn and Child Health and Development Team, Department of Child and Adolescent Health and Development, \\ World Health Organization, 20 Avenue Appia, I21 I, Geneva, Switzerland
}

\begin{abstract}
The study was conducted to analyze recent trends in the coverage of selected child-survival interventions. A systematic analysis of the coverage of six key child-health interventions in 29 African and Asian countries that had two recent demographic and health surveys - the latest one carried out in 2001 onwards and the immediately preceding survey conducted after 1990-was undertaken. A regression model was used for examining the relationship between the changes in the coverage of interventions and the changes in rates of mortality among children aged less than five years (under-five mortality). A limited increase in the coverage of key child-health interventions occurred in the past 5-10 years in these 29 countries in sub-Saharan Africa and Asia. More than half of the countries had no significant improvement or a significant reduction in the coverage of oral rehydration therapy (ORT) for diarrhoea (17/29) and care-seeking for acute respiratory infection (ARI) (16/29). Results of multivariate analysis revealed that increases in the coverage of early initiation of breastfeeding, ORT for diarrhoea, and care-seeking for ARI were significantly associated with reductions in under-five mortality. The results of this analysis should serve as a wake-up call for policymakers and programme managers in countries, donors, and international agencies to accelerate efforts to increase the coverage of key child-survival interventions. The following three main actions are proposed: setting of the clear target; mobilization of resources for increasing skilled birth attendants and health workers trained in integrated management of childhood illness; and implementation of community-based approaches.
\end{abstract}

Key words: Child health; Child survival; Infant health; Infant survival; Interventions; Asia; Africa

\section{INTRODUCTION}

The first decade of the new millennium has seen a renewed interest in child survival and achievement of the Millennium Development Goal 4 (MDG 4) of reducing the under-five mortality rate by twothirds between 1990 and 2015 (1). The World Health Report 2005 highlighted a mixed pattern of progress towards the achievement of MDG 4: 93 countries were reported to be 'on-track'; another 51 countries were showing slow progress; 29 countries had 'stagnating' mortality rates; and 14 countries

Correspondence and reprint requests should be addressed to:

Dr. Rajiv Bahl

Department of Child and Adolescent Health and

Development

World Health Organization

20 Avenue Appia, 1211 Geneva

Switzerland

Email: bahlr@who.int

Fax: +41227914853 showed increased mortality rates since 1990 (2). The Child Survival Countdown initiative assessed 68 countries selected based on their high burden of maternal and child mortality (3), most of which are in sub-Saharan Africa and Asia-the two regions which together account for over $75 \%$ of the world's child deaths (4). Of these, only 16 countries were considered as being 'on-track' (3). A recent report by the United Nations Children's Fund (UNICEF) has also shown that insufficient or no progress towards MDG 4 has been seen in sub-Saharan Africa and in South Asia (5).

Most child deaths occur due to a small number of conditions that, even in the poorest settings, could be mostly prevented with a small number of interventions that are well-known, feasible, affordable, and deliverable without complex technology. Globally, child mortality could be reduced substantially if all children in the world received a small set of effective interventions, which include, among others, 
oral rehydration therapy (ORT), breastfeeding, and antibiotics for pneumonia $(6,7)$. However, despite the renewed interest in improving child survival seen in recent years, their current coverage remains critically low (8).

There is sound evidence that a set of about 20 effective interventions could reduce child mortality by more than $60 \%$ if they reached the universal coverage (6). Increasing the coverage of such interventions is essential for the achievement of MDG 4. Systematic and periodic analyses of trends in the coverage of key child-survival interventions are crucial for monitoring progress at the country level and identifying countries that are making consistent progress and those that need additional assistance. Such an analysis could pave the way for a more comprehensive examination of the country situation, potentially leading to more focused actions and, therefore, contributing to make the universal coverage a reality.

We, therefore, undertook a systematic analysis of the coverage of selected neonatal and child-health interventions in sub-Saharan Africa and Asian countries that had had two recent demographic and health surveys. We also examined the association of trends in the coverage of these interventions with changes in the rates of under-five mortality.

\section{MATERIALS AND METHODS}

We performed an ecological study in which countries were the unit of analysis. This study was based on the analysis of data from nationally-representative population surveys conducted at two points in time.

\section{Source of data}

Information on rates of under-five mortality and on the coverage of interventions was abstracted from the demographic and health surveys (DHSs), which are nationally-representative household surveys with large sample sizes that provide data on demographic and health indicators and that are generally carried out every 3-5 years (9).

\section{Selection of countries}

We included all 29 countries-21 in sub-Saharan Africa and eight in Asia-which had had two DHSs - the most recent in 2001 onwards (20012006) and immediately previous one (baseline) conducted after 1990 (1990-2001).
Selected interventions and definition of indicators

We selected three types of interventions for this analysis: neonatal health interventions (indicators: skilled care at birth, early initiation of breastfeeding), preventive child-health interventions (indicators: exclusive breastfeeding during the first six months of age, complete immunization of children aged 12-23 months), and curative child-health interventions [indicators: care-seeking for children with acute respiratory infection (ARI), and provision of oral rehydration therapy (ORT) for children with diarrhoea]. Information on child-health indicators was available for all the selected countries and on neonatal health interventions for 28 of 29 countries.

\section{Definitions of indicators}

Under-five mortality rates-probability of dying before the fifth birthday (5q0) for the five-year period before the survey, according to the conventional life-table approach and expressed per 1,000 livebirths.

Skilled care at birth-the percentage of livebirths in the last three years preceding the survey attended by a skilled service provider. Although the definition of skilled service provider varies by country, it usually includes doctors, nurses, midwives, and medical assistants/clinical officers and excludes traditional birth attendants.

Early initiation of breastfeeding - the percentage of children born in the three years preceding the survey, who started breastfeeding within one hour of birth.

Exclusive breastfeeding of infants aged less than six months - the percentage of living children aged less than six months, who are exclusively breastfed.

Complete immunization of children at age 12 23 months-the percentage of children aged 12-23 months, who had received all recommended vaccinations (vaccination against tuberculosis-BCG, three doses each of DPT and polio vaccines, and a measles vaccine) at any time before the survey.

Oral rehydration therapy (ORT) for children with diarrhoea-the percentage of children, aged less than three years, with diarrhoea in the two weeks preceding the survey, who received oral rehydration solution or a recommended home solution. 
Care-seeking for children with ARI-the percentage of children, aged less than three years, with a cough accompanied with rapid breathing or with fever during the two weeks preceding the survey for whom care was sought from an appropriate health facility or service provider.

\section{Analysis}

We calculated the absolute and percentage average yearly changes in under-five mortality rates for all the 29 countries-21 African countries and eight Asian countries-between the most recent and the previous DHS. We assessed yearly changes to standardize for the inter-survey periods, which varied from four years in Malawi and Nigeria to 15.5 years in Pakistan and to allow inter-country comparisons.

Absolute changes in under-five mortality rates were computed by dividing the difference in the mortality rates between the most recent and the baseline survey by the number of years between the two surveys. The percentage changes in underfive mortality rates were calculated by dividing the difference in under-five mortality rates between the two surveys by the baseline mortality and then dividing by the number of years between the two surveys.

We also assessed the changes in under-five mortality between the baseline DHS (1990-2001) and the previous DHS (1987-1996) to compare with the changes observed in the most recent period. Data were available for 21 of the 29 countries.
We calculated the absolute yearly changes in coverage for each of the six interventions and the 95\% confidence intervals of these changes using the Stata software (version 9.0) (10).

We summarized the changes in under-five mortality and intervention coverage as medians and interquartile ranges. Finally, we explored the association of the change in the coverage of each intervention with the change in under-five mortality using multiple regression analysis in Stata.

\section{RESULTS}

Of the 29 selected countries, 21 are in sub-Saharan Africa (Benin, Burkina Faso, Cameroon, Chad, Eritrea, Ethiopia, Ghana, Guinea, Kenya, Madagascar, Malawi, Mali, Mozambique, Niger, Nigeria, Rwanda, Senegal, Tanzania, Uganda, Zambia, and Zimbabwe) and 8 in Asia (Bangladesh, Cambodia, India, Indonesia, Nepal, Pakistan, The Philippines, and Viet Nam). Together, these 29 countries account for $63 \%$ of global child deaths. There was a $3.3 \%$ annual reduction in under-five mortality between the two most recent DHSs, somewhat higher in Asia (3.9\% per year) than in sub-Saharan Africa (3.2\% per year) (Table 1$)$.

Information on reduction in mortality between the two surveys before the most recent one was used for comparing the changes in mortality rates described above with the changes during an earlier period. Between the two surveys before the most recent one, there had been a median increase of $0.6 \%$ per year in 16 coun-

\begin{tabular}{|lcc|}
\hline Table 1. Median annual changes in under-five mortality \\
\hline Indicator & $\begin{array}{c}\text { Median (IQR) annual } \\
\text { change between the most } \\
\text { recent and the previous DHS } \\
\text { (No. of countries: 29- } \\
21 \text { in Africa, 8 in Asia) }\end{array}$ & $\begin{array}{c}\text { Median (IQR) annual } \\
\text { change between 2 DHS be- } \\
\text { fore the most recent one } \\
\text { (No. of countries: 21- } \\
\text { 16 in Africa, 5 in Asia) }\end{array}$ \\
\hline $\begin{array}{l}\text { Annual change in under-five mortality } \\
\text { rate (under-five deaths/1,000 livebirths) }\end{array}$ & $-4.0(-7.6,-1.7)$ & $-1.2(-5.5,3.2)$ \\
Overall & $-6.1(-8.1,-2.2)$ & $1.1(-1.7,3.4)$ \\
Africa & $-2.6(-4.5,-1.5)$ & $-5.4(-7.2,-2.5)$ \\
Asia & & \\
\% of annual change in under-five & & $-0.6(-2.3,2.5)$ \\
mortality (as a percentage of mortality & & $0.6(-1.1,2.9)$ \\
at baseline) & $-3.3(-4.5,-1.4)$ & $-4.6(-6.3,-2.2)$ \\
Overall & $-3.2(-4.5,-1.3)$ & \\
Africa & $-3.9(-6.6,-2.2)$ & \\
Asia & & \\
\hline DHS=Demographic and health survey; IQR=Interquartile range & \\
\hline
\end{tabular}


tries in Africa from which these data were available, and a $4.6 \%$ median reduction per year in five countries in Asia (Table 1). Restricting the analysis of the most recent period to these 21 countries, the corresponding median reductions were $3.2 \%$ per year in sub-Saharan Africa and $3.5 \%$ per year in Asia.

The figure shows the annual changes in the cov- erage of the six selected interventions with respective confidence intervals. More than half of the countries had no significant improvement or had a reduction in the coverage of ORT for diarrhoea $(17 / 29)$ and care-seeking for ARI (16/29). On the other hand, only one-fifth of countries had a $2 \%$ or greater increase in the coverage of ORT (6/29) and roughly a quarter of countries in care-seeking for ARI $(8 / 29)$.
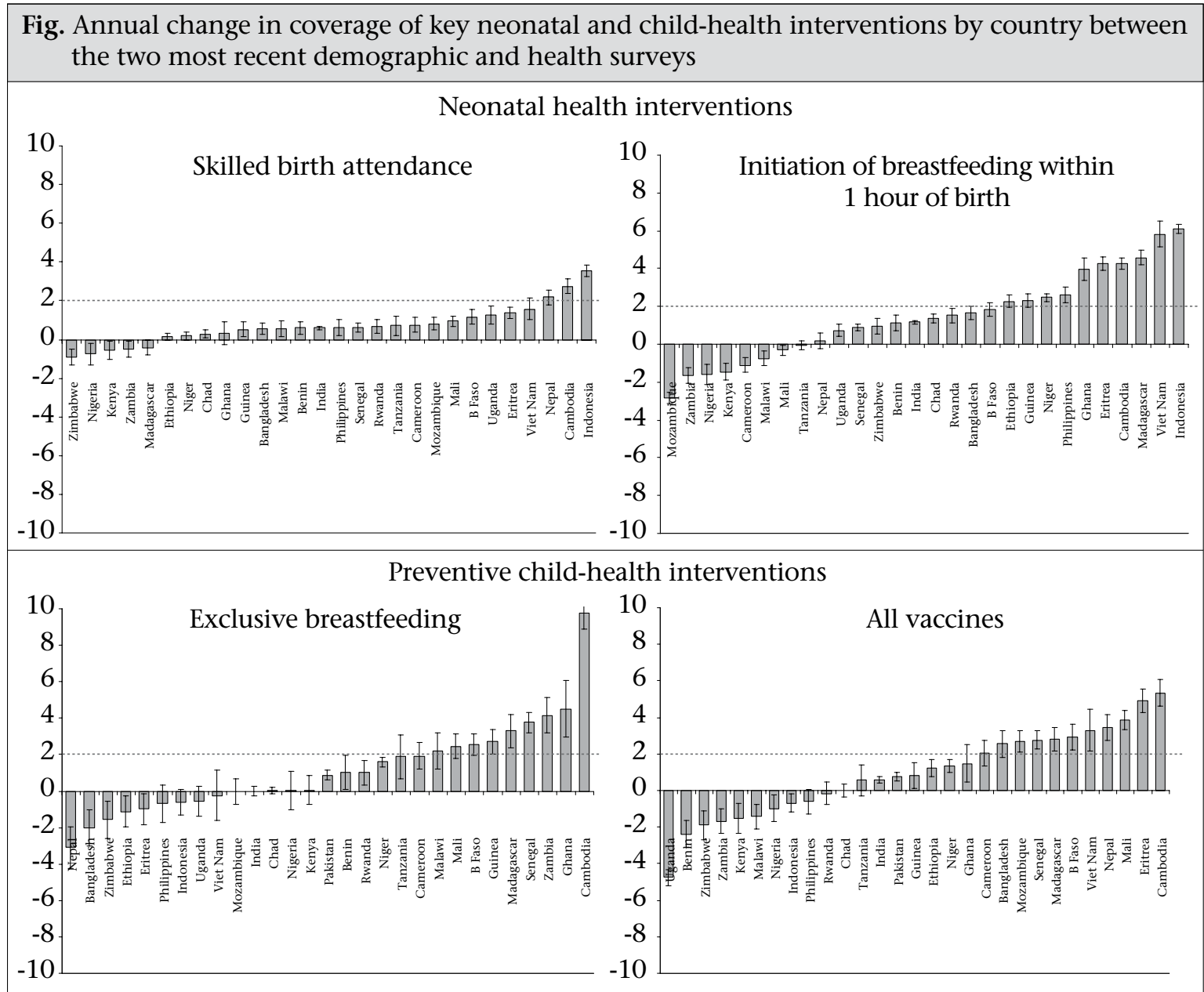

Curative child-health interventions
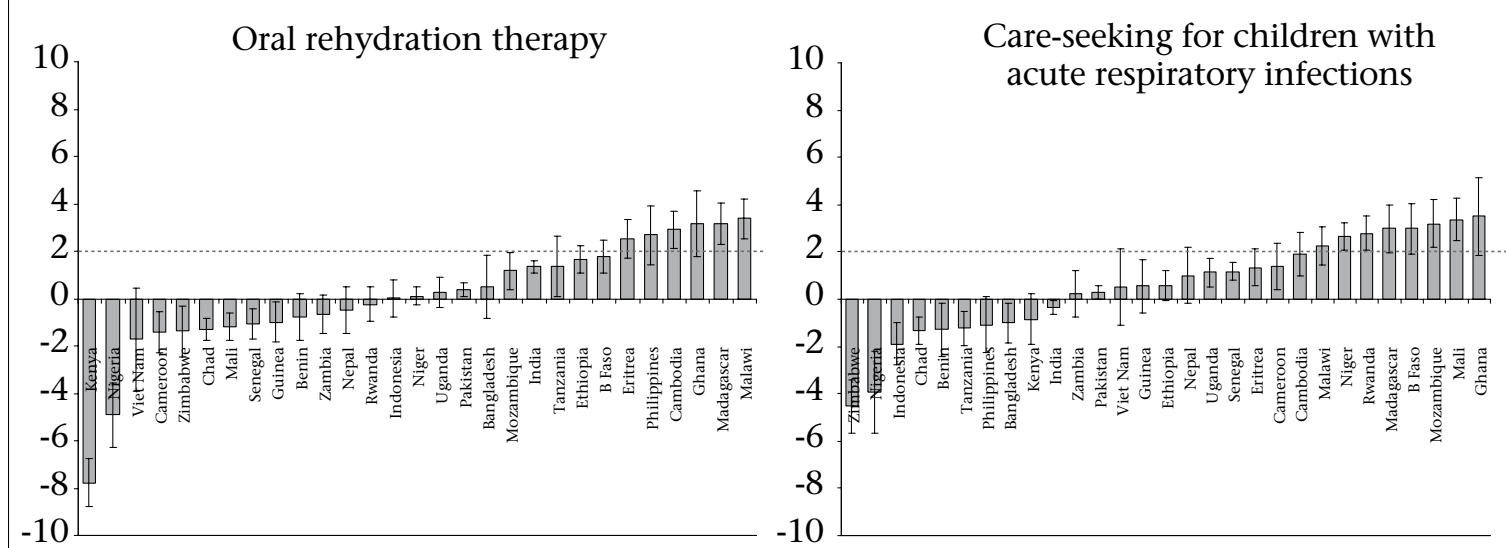
At the first survey assessment, the median coverage of exclusive breastfeeding among 0-6 months old children was $31 \%$ ( $n=29$ countries) and that of full vaccination among 12-23 months old children was $42 \%$ ( $n=29$ countries). Approximately half of the countries had either a reduction or no improvement in exclusive breastfeeding (14/29) and vaccination $(12 / 29)$ coverage. In contrast, about one-third of the countries had a significant increase in the coverage of exclusive breastfeeding (9/29) and of complete immunization $(11 / 29)$ of $2 \%$ or greater.

At baseline, the median coverage of skilled care at birth was $42 \%$ ( $n=29$ countries), Between the two survey periods, a $10 \%$ increase in the coverage resulted in a median $46 \%$ skilled birth attendance at the end-point survey. Eight of the 29 countries showed either a reduction or no improvement in the coverage of skilled care at birth and nine of the 29 countries in early initiation of breastfeeding. Although 10 of the 29 countries had a significant increase in the coverage of early initiation of breastfeeding of $2 \%$ or greater, only three countries could achieve this level of the increased coverage for skilled care at birth.

An interesting relationship between the baseline coverage and the change in coverage between the last two surveys was observed. Countries with very low baseline coverage $(<30 \%)$ of ORT for diarrhoea, care-seeking for ARI, exclusive breastfeeding, and early initiation of breastfeeding made greater progress in increasing coverage than those with relatively higher baseline coverage (>60\%). The latter showed mostly no progress or reduction in coverage (data not shown). This pattern was not observed for changes in skilled care at birth and vaccination coverage.

Table 2 summarizes the coverage of key childhealth interventions at baseline and the annual median changes in coverage by region. At baseline, the coverage of neonatal health interventions was higher in sub-Saharan Africa compared to the Asian countries, with a 2.2-fold difference in early initiation of breastfeeding. Conversely, the coverage of all preventive and curative child-health interventions was higher in the Asian countries.

In sub-Saharan Africa, the greatest increase in annual coverage was observed for exclusive breastfeeding and care-seeking for ARI. ORT for diarrhoea and skilled care at birth had the worst performance. In Asia, the greatest increase in coverage was observed for skilled care at birth, early initiation of breastfeeding, and vaccination. There was no or negligible change in the coverage of exclusive breastfeeding, ORT for diarrhoea, and care-seeking for ARI.

Table 3 shows the association of changes in the coverage of the six selected interventions with changes in under-five mortality between the two DHSs, adjusted for baseline under-five mortality, baseline coverage, and region. Increases in the coverage of early initiation of breastfeeding, ORT for diarrhoea, and care-seeking for ARI were associated with significant reductions in under-five mortality. The relationship shown between these three interventions and under-five mortality translates into a reduction of 1.2-1.5 under-five deaths per 1,000 livebirths for each 1\% increase in the coverage of any of these interventions.

\begin{tabular}{|c|c|c|c|c|}
\hline \multirow{2}{*}{ Intervention } & \multicolumn{2}{|c|}{$\begin{array}{l}\text { Sub-Saharan Africa } \\
(\mathrm{n}=21 \text { countries })\end{array}$} & \multicolumn{2}{|c|}{$\begin{array}{c}\text { Asia }(\mathrm{n}=8 \text { countries }) \\
(\mathrm{n}=7 \text { for intervention for } \\
\text { newborns })\end{array}$} \\
\hline & $\begin{array}{l}\text { Median base- } \\
\text { line coverage }\end{array}$ & $\begin{array}{l}\text { Median (IQR) } \\
\text { annual chang- } \\
\text { es in coverage }\end{array}$ & $\begin{array}{l}\text { Median } \\
\text { baseline } \\
\text { coverage }\end{array}$ & $\begin{array}{c}\text { Median (IQR) } \\
\text { annual changes } \\
\text { in coverage }\end{array}$ \\
\hline Skilled care at birth & 43 & $0.5(0.1,0.8)$ & 38 & $1.6(0.6,2.7)$ \\
\hline Breastfed within 1 hour of birth & 38 & $0.9(-0.8,2.3)$ & 17 & $2.6(1.2,5.8)$ \\
\hline$<6$-month olds exclusively breastfed & 30 & $1.6(0,2.6)$ & 40 & $-0.4(-1.4,0.4)$ \\
\hline $\begin{array}{l}\text { Received all vaccines, aged } 12-23 \\
\text { months }\end{array}$ & 41 & $0.8(-1.4,2.1)$ & 53 & $1.7(0,3.4)$ \\
\hline Received ORT during diarrhoea & 34 & $-0.2(-1.2,1.6)$ & 43 & $0.4(-0.3,2.0)$ \\
\hline Sought care for ARI & 37 & $1.4(-0.9,2.8)$ & 63 & $0(-1.0,0.8)$ \\
\hline \multicolumn{5}{|c|}{$\begin{array}{l}\text { ARI=Acute respiratory infection; DHS=Demographic and health survey; IQR=Interquartile range; } \\
\text { ORT=Oral rehydration therapy }\end{array}$} \\
\hline
\end{tabular}




\begin{tabular}{|c|c|}
\hline Intervention & $\begin{array}{l}\text { Adjusted }{ }^{*} \text { change in under-five mortality (per } \\
1,000 \text { livebirths) associated with } 1 \% \text { change in } \\
\text { coverage }(95 \% \mathrm{CI})\end{array}$ \\
\hline Skilled care at birth $(\mathrm{n}=28)$ & $-1.6(-4.0,0.8)$ \\
\hline Breastfed within 1 hour of birth $(n=28)$ & $-1.5(-2.3,-0.6)$ \\
\hline Exclusive breastfeeding, $<6$ months old $(n=29)$ & $-0.6(-1.4,0.2)$ \\
\hline Received all vaccines, $12-23$ months old $(\mathrm{n}=29)$ & $-0.6(-1.4,0.2)$ \\
\hline Received ORT during diarrhoea $(\mathrm{n}=29)$ & $-1.2(-1.9,-0.5)$ \\
\hline Sought care for ARI $(\mathrm{n}=29)$ & $-1.2(-2.2,-0.2)$ \\
\hline
\end{tabular}

\section{DISCUSSION}

The main finding of this analysis is that a very little increase in the coverage of key neonatal and childhealth interventions occurred in the past 5-10 years in countries in sub-Saharan Africa and Asia. These improvements have been smaller in countries that already had a moderately-high coverage at baseline. Based on the observed median increase in coverage, it would take a minimum of 28 years in Asia and 44 years in sub-Saharan Africa to reach universal coverage for any one of the included interventions.

While it is heartening to see that the percent annual rate of reduction in under-five mortality in Africa increased in the recent years compared to that observed in the 1990s, the current reductions would not be sufficient to achieve MDG 4. Countries in Asia had almost the same percentage of the annual rate of decline in mortality in 1990s and 2000s. Data were available for the earlier period for nearly three-quarters of countries, and findings were almost the same if analysis of the recent period were restricted to only these countries. An assessment of progress of the countries in child survival has shown that only six (Bangladesh, Eritrea, Indonesia, Nepal, The Philippines, and Viet Nam) of the 29 countries included in our review seem to be well-positioned to achieve MDG 4.

The universal coverage of available interventions could prevent about two-thirds of childhood deaths, and two single interventions-ORT and exclusive breastfeeding — have been each estimated to avert more than $10 \%$ of all deaths of under-five children (6). The slowing down in progress of reduction in mortality in Asia could be, at least in part, the result of stagnation or relative reductions of some main child-health interventions, including exclusive breastfeeding, ORT, and also care-seeking for children with ARI in at least half of the eight countries examined. Many Asian countries included in this analysis have been recently experiencing important economic growth. However, such development has not equally benefited the whole population, and some of these countries are subjected to enormous health gaps. Inequities in provision and access to care and in subsequent outcomes are likely to be reflected in our average measures. Conversely, sub-Saharan Africa has been lately conferring increased attention to child survival.

The results of our ecological analysis provided further evidence that increases in the coverage of key interventions were associated with reductions in under-five mortality. Acceleration of specific efforts for improving the coverage of key interventions is, therefore, a plausible way for health programmes to contribute to the achievement of MDG 4. The decrease in coverage has been seen as associated with the increase in mortality. Of the seven countries that had an increase in under-five mortality between 1990 and 2005 (11), six had some of the highest decreases in the coverage of ORT, and three had substantial reductions in the coverage of careseeking for children with ARI in the same period. On the other hand, in Eritrea, one of the few African countries that are likely to achieve MDG 4, an accelerated $2.5 \%$ annual increase in the coverage of ORT for children with diarrhoea was observed. Although in a smaller extent, annual increases in the coverage of skilled care at birth and of care-seeking for children with ARI have also been detected in the country.

The lack of progress in the coverage of ORT for children with diarrhoea, similarly low in both the regions, and in care-seeking for children with ARI is most striking. It is even more disturbing to learn that about a quarter of the countries showed a re- 
cent reduction in the coverage of these interventions. Using a slightly-different definition of ORT (oral rehydration solution, recommended home solution or increased fluids) from ours, Ram et al. have recently shown very similar results (12). Increase in the coverage of skilled care at birth has been slow in all but three countries. However, these three interventions fall in the category of 'clinical care' rather than 'outreach' or 'population-based' interventions. While this may reflect some inherent problems in scaling-up of clinical care interventions, we believe that these interventions have received substantially less attention and support from the global public-health community. Zambia, for example, achieved a $4 \%$ annual increase in the percentage of children who started breastfeeding within one hour of birth. In contrast, it has shown an annual 1\% decrease in the coverage of ORT for children with diarrhoea and a stagnated coverage in care-seeking for children with ARI.

We decided to use data only from the DHSs for analyses presented in this paper. We appreciate that data from surveys such as DHSs have inherent limitations, which include recall bias, reporting bias, and the fact that indicators for the coverage of child-health interventions are reported only for living children. However, these surveys are perhaps the best available source of representative, population-based coverage and information on mortality from developing countries with weak health systems and almost non-existent vital registration data. Another source of similar data is the Multiple Indicators Cluster Surveys, of which three rounds have been conducted, the last one in 2005-2007. However, data from both round 2 and 3 are currently in the public domain for only six countries from sub-Saharan Africa and Asia.

There are some limitations of this analysis that merit consideration. We used an ecological design, which we believe is appropriate for understanding the big picture of trends in the coverage of neonatal and child-health interventions but cannot establish a causal relationship between the coverage and the reduction in under-five mortality. As the DHSs are not carried out in all countries, we could include only 29 countries in our analysis. However, these 29 countries account for 6.1 million annual underfive deaths, i.e. $63 \%$ of all childhood deaths worldwide and more than $70 \%$ of under-five deaths each in sub-Saharan Africa and Asia. Finally, the most recent data in our analysis were collected between 2001 and 2006 (median 2004). To look for more recent changes in the intervention coverage, we examined separately data from countries in which the most recent survey was carried out in 20052006. These changes were similar to those in the overall analysis.

We propose three specific actions that could help promote increase in the coverage of key neonatal and child-health interventions. First, every country should set clear targets for increasing the coverage and monitor its progress every 2-3 years. Based on the progress made by some countries and on reductions in mortality associated with an increased coverage, we propose that the target should be at least $2 \%$ annual increase in coverage for each key intervention. Second, countries should mobilize more resources to have skilled birth attendants and health workers trained in integrated management of childhood illness. Finally, countries should consider undertaking interventions with innovative approaches, including community-based management of childhood diseases, to increase the coverage of interventions $(13,14)$. Examples of such approaches are visits to home for care of newborns during the first week of life and case management of pneumonia by community health workers. While community case management of pneumonia by community health workers has been proven to be an effective strategy to complement facilitybased case management $(15,16)$, and the policy statements by the WHO and UNICEF promoting community management of pneumonia exist, only about one-third of the 29 countries included in this analysis (Bangladesh, Benin, India, Indonesia, Mali, Niger, Pakistan, Uganda, and Zambia) have permissive policies and implemented community case management of pneumonia by community health workers at some scale (17).

In conclusion, this analysis should serve as a wakeup call for policy-makers and programme managers in countries, donors, and international agencies to set clear coverage targets and implement effective strategies and monitor progress to achieve them.

\section{ACKNOWLEDGEMENTS}

The authors thank Kim Mulholland for critically reviewing the final draft and providing important comments and suggestions.

\section{REFERENCES}

1. United Nations Millennium Development Goals. (http://www.un.org/millenniumgoals, accessed on 7 March 2008).

2. World Health Organization. World health report 2005: make every mother and child count. Geneva: World Health Organization, 2005:12-3. 
3. United Nations Children's Fund. Countdown to 2015: tracking progress in maternal, newborn and child survival. The 2008 report. New York, NY: United Nations Children's Fund, 2008:18-9.

4. Bryce J, Boschi-Pinto C, Shibuya K, Black RE; WHO Child Health Epidemiology Reference Group. WHO estimates of the causes of death in children. Lancet 2005;365:1147-52.

5. United Nations Children's Fund. Progress for children: a world fit for children statistical review. New York, NY: United Nations Children's Fund, 2007:18-9.

6. Jones G, Steketee RW, Black RE, Bhutta ZA, Morris SS; Bellagio Child Survival Study Group. How many child deaths can we prevent this year? Lancet 2003;362:65-71.

7. Darmstadt GL, Bhutta ZA, Cousens S, Adam T, Walker N, de Bernis L; Lancet Neonatal Survival Steering Team. Evidence-based, cost-effective interventions: how many newborn babies can we save? Lancet 2005;365:977-88.

8. Bryce J, Terreri N, Victora CG, Mason E, Daelmans B, Bhutta ZA et al. Countdown to 2015: tracking intervention coverage for child survival. Lancet 2006;368:1067-76.

9. MEASURE DHS (Demographic and health surveys) project. (http://www.measuredhs.com/, accessed on 20 July 2008).

10. StataCorp. Stata statistical software SE: release 27.04.2005. College Station, TX: Stata Corporation, 2005.
11. United Nations Children's Fund. The state of the world's children 2007: women and children; the double dividend of gender equality. New York, NY: United Nations Children's Fund, 2006:138-41.

12. Ram PK, Chi M, Blum LS, Wamae AW, Mintz ED, Bartlett AV. Declines in case management of diarrhoea among children less than five years old (letter). Bull World Health Organ 2008,86:e-f.

13. Bryce J, Victora CG, Habicht JP, Black RE, Schpbier RW. Programmatic pathways to child survival: results of a multi-country evaluation of Integrated Management of Childhood Illness. Health Policy Plan 2005;20(Suppl 1);i5-i17.

14. Dawson P, Pradhan Y, Houston R, Karki S, Poudel D, Hodgins S. From research to national expansion: 20 years' experience of community-based management of childhood pneumonia in Nepal. Bull World Health Organ 2008;86:339-43.

15. Hadi A. Diagnosis of pneumonia by community health volunteers: experience of BRAC, Bangladesh. Trop Doct 2001;31:75-7.

16. Menhaz A, Billoo AG, Yasmeen T, Nankani K. Detection and management of pneumonia by community health workers-a community intervention study in Rehri village, Pakistan. J Pak Med Assoc 1997;47:42-5.

17. Marsh DR, Gilroy KE, Van de Weerdt R, Wansi E, Qazi S. Community case management of pneumonia-at a tipping point? Bull World Health Organ 2008;86:381-9. 